\title{
GENERALIZED RODRIGUES FORMULA SOLUTIONS FOR CERTAIN LINEAR DIFFERENTIAL EQUATIONS
}

BY

JAMES M. HORNER

In [1] and [2] solutions were given in terms of generalized Rodrigues formulas for the second order differential equation

$$
P_{2}(x) y^{\prime \prime}+P_{1}(x) y^{\prime}+P_{0} y=R(x),
$$

where $P_{i}(x)$ is a polynomial of degree not exceeding $i$. These results depended on roots of the associated quadratic equation

$$
\frac{1}{2} t(t+1) P_{2}^{\prime \prime}-t P_{1}^{\prime}+P_{0}=0
$$

It was found that, when this equation has a positive integer root $n$, (1) has solutions which can be expressed in terms of a generalized Rodrigues formula having $n-1$ for the index of differentiation. For nonpositive integer roots of (2) a general solution for (1) can be given by an iterated indefinite integral and for nonintegral roots the corresponding solution is a contour integral which reduces to the Rodrigues formula for integer roots. The contour integral result was obtained only for the homogeneous form of (1).

The purpose of this paper is to present the remaining results obtained by the author in [3], namely the extension of the above results for secondorder equations to certain $n$ th-order linear differential equations. The equation to be discussed is

$$
L[y]=\sum_{i=0}^{n} P_{i}(x) y^{(i)}=R(x), \quad n \geqq 2,
$$

where $P_{i}(x)$ is a polynomial of degree $\leqq i$ and, except for Theorem $6, x$ is a real variable. It is assumed that $P_{0} \neq 0$ and that $P_{n}(x) \not \equiv 0$.

Consider now the system of equations

$$
F_{r}(t)\left\{\frac{r(t+1)}{(r+1)} P_{n}^{(r+1)}-P_{n-1}^{(r)}\right\}+P_{n-r-1} \equiv x 0,
$$

where $F_{r}(t)$ is the polynomial of degree $r$ given by

$$
F_{r}(t)=t(t-1)(t-2) \cdots(t-r+1) / r !
$$

and $r$ takes the values $r=1,2,3, \cdots, n-1$.

Received by the editors December 9, 1963. 
Notice that the identity in (4) is with respect to $x$. That is, the system of equations (4) consists of polynomials in $t$ arising by equating the coefficient of each power of $x$ to zero. For each value of $r$ the system (4) produces $n-r$ polynomials of degree $r+1$ in $t$. So the total system consists of $\frac{1}{2} n(n-1)$ polynomials; one of degree $n$, two of degree $n-1$, three of degree $n-2, \cdots$, and finally $n-1$ polynomials of degree two.

In the following discussion only those differential equations (3) whose polynomial coefficients satisfy (4) are considered. The system (4) of polynomials in $t$ is called the associated system and it is assumed that not all of the polynomials in the associated system are identically zero. If those equations of the associated system which are not identically zero have a common root it is called a root of the associated system. We will consider only those differential equations for which the associated system has at least one root. Notice that for $n=2$ the associated system reduces to the single equation (2).

The restriction $P_{0} \neq 0$ prohibits the roots $t=0,1,2, \cdots, n-2$ and also requires that either $P_{n}(x)$ or $x P_{n-1}(x)$ be exactly of degree $n$. It is important to note that it is always possible to exhibit a differential equation of the form (3) whose associated system has a prescribed root (with the exceptions $t=0,1,2, \cdots, n-2)$. A particular method is to select the desired root, choose $P_{n}(x)$ arbitrarily (but of degree $n$ ), take $P_{n-1}(x) \equiv 0$; and define the remaining polynomial coefficients by (4).

Actually the homogeneous form of the differential equation (3) with the restrictive condition that the associated system has at least one root yields precisely the general Pochhammer equation [4, pp. 109-113]. To see this, put $R(x) \equiv 0$ in (3), suppose that the associated system (4) has the root $t=-u-1$, and let $G(x)=-P_{n-1}(x)-u P_{n}^{\prime}(x)$. Then (4) becomes

$$
\begin{aligned}
P_{n-r-1}(x)= & (-1)^{r+1} \frac{u(u+1) \cdots(u+r)}{(r+1) !} P_{n}^{(r+1)}(x) \\
& +(-1)^{r+1} \frac{(u+1)(u+2) \cdots(u+r)}{(r+1) !} G^{(r)}(x),
\end{aligned}
$$

for $r=1,2,3, \cdots, n-1$, and so (3) can be written

$$
\begin{aligned}
L[y]= & P_{n} y^{(n)}-u P_{n}^{\prime} y^{(n-1)}+\frac{u(u+1)}{2 !} P_{n}^{\prime \prime} y^{(n-2)}-\cdots \\
& -G y^{(n-1)}+\frac{(u+1)}{1 !} G^{\prime} y^{(n-2)}-\cdots=0 .
\end{aligned}
$$

(See also [5, pp. 454-465].)

It is well known that this equation can be solved using the Euler transformation [4, pp. 97-99], [5, pp. 191-193], [6, pp. 333-338]. This trans- 
formation gives rise to the so-called Jordan-Pochhammer contour integrals from which it is possible to obtain $n$ distinct solutions for (7) [7, pp. 240-276], $[8],[9],[10]$.

However, in the discussion which follows the form given by (3) and (4) is more convenient and solutions (including the Jordan-Pochhammer integrals) can be obtained for both (3) and the homogeneous form of (3) without appealing directly to the Euler transformation.

For convenience, let

$$
W(x)=\exp \left[-\int\left(P_{n-1} / P_{n}\right) d x\right]
$$

and

$$
g(p, x)=\left[P_{n}(x)\right]^{p} W(x),
$$

where (except in Theorem 6) $p$ is an integer. $W(x)$ is, of course, a constant multiple of the Wronskian of any fundamental system of solutions for (3) $[11$, p. 328].

THEOREM 1. If $R(x) \equiv 0$ and the associated system has a positive integer root $j \geqq(n-1)$ then (3) is satisfied by the $j+1$ functions

$$
y_{1}(x)=\frac{d^{j-n+1}}{d x^{j-n+1}}\{g(j, x)\}
$$

and

$$
y_{2+i}(x)=\frac{d^{j-n+1}}{d x^{j-n+1}}\left\{g(j, x) \int\left[x^{i} / g(j+1, x)\right] d x\right\},
$$

where $i=0,1,2, \cdots, j-1$.

Proof. Construct the first-order differential equation

$$
P_{n} z^{\prime}+\left(P_{n-1}-j P_{n}^{\prime}\right) z=Q_{j-1}(x),
$$

where $Q_{j-1}(x)$ is an arbitrary polynomial of degree $\leqq(j-1)$.

By elementary methods (e.g. $[12$, p. 19]) or by direct substitution (12) is seen to have the general solution

$$
z=g(j, x)\left\{k+\int\left[Q_{j-1} / g(j+1, x)\right] d x\right\}
$$

where $k$ is an arbitrary constant.

Now, using the Leibnitz rule for product differentiation [13, p. 19], differentiate (12) $j$ times. This yields

$$
\sum_{k=0}^{j} \frac{j !}{k !(j-k) !} P_{n}^{(k)} z^{(j+1-k)}+\sum_{k=0}^{j} \frac{j !}{k !(j-k) !}\left\{P_{n-1}^{(k)}-j P_{n}^{(k+1)}\right\} z^{(j-k)}=0 .
$$


In the first sum remove the first term and in the remaining sum replace $k$ by $r+1$. In the second sum replace $k$ by $r$ and remove the last term. The two resulting sums can then be combined and (14) becomes

$$
\begin{aligned}
P_{n} z^{(j+1)} & +\left(P_{n-1}^{(j)}-j P_{n}^{(j+1)}\right) z \\
& +\sum_{r=0}^{j-1} \frac{j !}{r !(j-r) !}\left[P_{n-1}^{(r)}-\frac{r(j+1)}{(r+1)} P_{n}^{(r+1)}\right] z^{(j-r)}=0 .
\end{aligned}
$$

Now, in (15) remove the first term of the sum and replace the term corresponding to $r=j$. This gives

$$
\begin{aligned}
P_{n} z^{(j+1)} & +P_{n-1} z^{(j)} \\
& -\sum_{r=1}^{j} \frac{j !}{r !(j-r) !}\left[\frac{r(j+1)}{(r+1)} P_{n}^{(r+1)}-P_{n-1}^{(r)}\right] z^{(j-r)}=0 .
\end{aligned}
$$

Since the degree of the polynomials $P_{i}$ does not exceed the subscript, any terms in (16) for which $r>(n-1)$ are zero. So the upper limit on the sum can be changed to $n-1$. Also, from $(5), F_{r}(j)=j ! /[r !(j-r) !]$ so that, with the aid of (4), the coefficient of $z^{(j-r)}$ in (16) is seen to be $P_{n-r-1}$.

Hence, (16) is

$$
\sum_{k=0}^{n} P_{n-k} z^{(j+1-k)}=0 .
$$

But this is exactly (3) with $y$ replaced by $z^{(j-n+1)}$ when $R(x) \equiv 0$. Thus when $R(x) \equiv 0$ (3) is satisfied by

$$
y=\frac{d^{j-n+1}}{d x^{j-n+1}}\left[g(j, x)\left\{k+\int\left[Q_{j-1} / g(j+1, x)\right] d x\right\}\right]
$$

for every constant $k$ and every polynomial $Q_{j-1}(x)$ whose degree is $\leqq(j-1)$.

To obtain $y_{1}(x)$ take $k=1$ and $Q_{j-1} \equiv 0$. The remaining solutions $y_{2+i}(x)$ are obtained by taking $k=0$ and $Q_{j-1}=x^{i}$.

ln some cases it is convenient to replace $g(j, x)$ by some equivalent solution to the homogeneous form of (12).

For the particular case $n=2$ results somewhat similar to the first solution of Theorem 1 have been obtained by Brenke [14]. The method used by Brenke consisted essentially of showing that certain orthogonal polynomials, given in terms of a Rodrigues formula by Abramescu [15], satisfied a secondorder equation (see also [4, pp. 272-273]).

Corollary 2. If $P_{n}(x)$ is a polynomial of degree $n$ then the polynomials

$$
P_{k(n-1)}^{*}(x)=\frac{d^{k}}{d x^{k}}\left\{\left[P_{n}(x)\right]^{k}\right\}
$$

satisfy the nth-order differential equation 


$$
\sum_{i=0}^{n} \frac{(k+n-1) ![(n-1)-(i-1)(k+1)]}{(k+n-i) ! i !} P_{n}^{(i)} y^{(n-i)}=0 .
$$

Proof. The first term of (20) is $P_{n} y^{(n)}$ and, in the notation of (3), $P_{n-1}$ $=(n-1) P_{n}^{\prime}$ and

$$
P_{n-r-1} \equiv \frac{(k+n-1) ![n-1-r(k+1)]}{(k+n-r-1) !(r+1) !} P_{n}^{(r+1)} .
$$

Substituting these values into (4), using (5), and removing the nonzero factor $P_{n}^{(r+1)} /(r+1)$ !, we obtain the associated system

$$
\begin{aligned}
& t(t-1) \cdots(t-r+1)[r(t+1)-(n-1)(r+1)] \\
& \quad=(k+n-1)(k+n-2) \cdots(k+n-r)[r(n+k)-(n-1)(r+1)],
\end{aligned}
$$

which obviously has the root $t=n+k-1$.

Now put $j=n+k-1$ in Theorem 1 and replace $g(j, x)$ by the equivalent form $\left[P_{n}(x)\right]^{j-n+1}=\left[P_{n}(x)\right]^{k}$. The conclusion then follows using $y_{1}(x)$ in Theorem 1 .

Example 1. The Legendre polynomials [16, pp. 302-336]

$$
p_{k}(x)=\frac{1}{2^{k} k !} \frac{d^{k}}{d x^{k}}\left[\left(x^{2}-1\right)^{k}\right]
$$

satisfy the second-order differential equation

$$
\left(x^{2}-1\right) y^{\prime \prime}+2 x y^{\prime}-k(k+1) y=0 .
$$

Example 2. The Appell polynomials [15, p. 209]

$$
p_{2 k}=\frac{1}{k !} \frac{d^{k}}{d x^{k}}\left[x^{k}\left(1-x^{2}\right)^{k}\right]
$$

satisfy the third-order differential equation

$$
\begin{aligned}
x\left(1-x^{2}\right) y^{\prime \prime \prime} & +2\left(1-3 x^{2}\right) y^{\prime \prime} \\
& +3\left(k^{2}-1\right)(k+2) x y^{\prime}+2 k^{2}(k+1)(k+2) y=0 .
\end{aligned}
$$

CoROllary 3. If $Q_{n}(x)$ is a polynomial of degree $n$ and in the differential equation

$$
y^{(n)}+\sum_{i=1}^{n} \frac{(n+k-1) !}{(n+k-i) !} Q_{n}^{(i)} y^{(n-i)}=0,
$$

we put $y=u(x) \exp \left[-Q_{n}(x)\right]$, the resulting differential equation is satisfied by the polynomials

$$
u_{k(n-1)}(x)=\exp \left[Q_{n}(x)\right] \frac{d^{k}}{d x^{k}}\left\{\exp \left[-Q_{n}(x)\right]\right\}
$$


Proof. The first term of (20) is $y^{(n)}$ and in the notation of (3) $P_{n} \equiv 1$, $P_{n-1} \equiv Q_{n}^{\prime}$, and

$$
P_{n-r-1} \equiv \frac{(n+k-1) !}{(n+k-1-r) !} Q_{n}^{(r+1)} .
$$

As in Corollary 2, substitution of these values into (4) shows that the associated system has the root $j=n+k-1$. In this case

$$
g(j, x)=\exp \left[-Q_{n}(x)\right],
$$

so (27) is satisfied by

$$
y(x)=\frac{d^{k}}{d x^{k}}\left\{\exp \left[-Q_{n}(x)\right]\right\},
$$

and the conclusion follows.

EXAmpLe 3. If in Corollary $3 Q_{n}(x)=x^{2}$, we obtain the Hermite equation $[17$, pp. 187-199]

$$
u^{\prime \prime}-2 x u^{\prime}+2 k u=0
$$

satisfied by

$$
H_{k}(x)=(-1)^{n} \exp \left(x^{2}\right) \frac{d^{k}}{d x^{k}}\left[\exp \left(-x^{2}\right)\right] .
$$

The method employed in Theorem 1 can also be used to obtain a particular solution to (3) when $R(x) \not \equiv 0$.

TheOREM 4. If the associated system has a positive integer root $j \geqq(n-1)$ then a particular solution to (3) is

$$
y_{p}(x)=\frac{d^{j-n+1}}{d x^{j-n+1}}\left\{g(j, x) \int\left[I_{j}(x) / g(j+1, x)\right] d x\right\},
$$

where

$$
I_{j}(x)=\iint \cdots \int R(x)(d x)^{j} .
$$

Proof. The proof is exactly the same as that of Theorem 1 after replacing $Q_{j-1}(x)$ by $I_{j}(x)$ in (12) and replacing the general solution (13) by

$$
z_{p}=g(j, x) \int\left[I_{j} / g(j+1, x)\right] d x .
$$

Differentiation of the left member of (12) $j$ times will, as before, give the left member of (3) while differentiation of $I_{j}(x) j$ times yields $R(x)$.

Because of Theorem 1 it is sufficient to neglect any polynomial terms of degree $\leqq(j-1)$ occurring in $I_{j}(x)$ before computing $y_{p}(x)$. In particu- 
lar, the constants of integration may be taken as zero when computing $I_{j}(x)$. If not, the effect is to combine with a particular solution some of the solutions in (10) and (11) for the homogeneous form of (3).

Example 4. The nonhomogeneous Legendre equation

$$
\left(x^{2}-1\right) y^{\prime \prime}+2 x y^{\prime}-k(k+1) y=e^{x}
$$

yields an associated system consisting of a single quadratic equation whose roots are $t=-k$ and $t=k+1$. So when $k$ is a non-negative integer a particular solution of (37) is

$$
y_{p}(x)=\frac{d^{k}}{d x^{k}}\left\{\left(x^{2}-1\right)^{k} \int e^{x}\left(x^{2}-1\right)^{-k-1} d x\right\} .
$$

In some instances it is desirable to replace the indefinite integrals by definite integrals. For example, $W(x)$ might be replaced by $W\left(x, x_{0}\right)$ where

$$
W\left(x, x_{0}\right)=\exp \left\{-\int_{x_{0}}^{x}\left[P_{n-1}(v) / P_{n}(v)\right] d v\right\},
$$

and $x_{0}$ is any point for which the integral exists and satisfies the necessary differentiability and integrability conditions implied in the conclusions of the theorems. In particular, $I_{j}(x)$ of the previous theorem may be replaced by

$$
I_{j}(x)=\int_{a_{j}}^{x} \int_{a_{j-1}}^{v} \ldots \int_{a_{1}}^{v} R(v)(d v)^{j}
$$

or, taking $a_{1}=a_{2}=\cdots=a_{j}=a$, by

$$
I_{j}(x)=\frac{1}{(j-1) !} \int_{a}^{x}(x-v)^{j-1} R(v) d v .
$$

The idea of interpreting iterated integrals as derivatives of negative order suggests the following result.

THEOREM 5. If the associated system has a negative integer root $t=-j$ and $R(x) \in C^{j}$ then every solution of (3) can be obtained from

$$
y(x)=\iint \cdots \int g(-j, x)\left\{k+\int\left[R^{(j)} / g(-j+1, x)\right] d x\right\}(d x)^{n+j-1}
$$

for appropriate choices of $k$ and the constants of integration.

Proof. By reversing the sum in (3) it becomes

$$
\sum_{k=0}^{n} P_{n-k} y^{(n-k)}=R(x) .
$$

Suppose that $y(x)$ is any solution to (43) and differentiate (43) $j$ times. This gives 


$$
\sum_{k=0}^{n} \sum_{i=0}^{j} \frac{j !}{i !(j-i) !} P_{n-k}^{(i)} y^{(n+j-k-i)}=R^{(j)} .
$$

Collecting coefficients of $y^{(n+j-q)}$ we have

$$
\sum_{q=0}^{n+j}\left(\sum_{i+k=q} \frac{j !}{i !(j-i) !} P_{n-k}^{(i)}\right) y^{(n+j-q)}=R^{(j)}, \quad i \leqq j, k \leqq n .
$$

Now remove the terms corresponding to $q=0$ and $q=1$ from the sum in (45). Also since $P_{n-k}^{(i)}=0$ if $i+k>n$ the upper limit on the first sum can be changed to $n$. Then (45) becomes

$$
P_{n} y^{(n+j)}+\left(P_{n-1}+j P_{n}^{\prime}\right) y^{(n+j-1)}
$$

$$
+\sum_{q=2}^{n} \sum_{i+k=q} \frac{j !}{i !(j-i) !} P_{n-k}^{(i)} y^{(n+j-q)}=R^{(j)}, \quad i \leqq j .
$$

So we are led to consider

$$
f_{q}=\sum_{i+k=q} \frac{j !}{i !(j-i) !} P_{n-k}^{(i)}, \quad 0 \leqq i \leqq j, q=2,3, \cdots, n .
$$

There are two cases to consider: those $q$ for which $q \leqq j$ and those for which $q>j$. If $q \leqq j$ then in (47) put $i=q-k$ in the summand and sum on $k$ from 0 to $q$. After a shift in summation index, (47) then becomes

$$
\begin{aligned}
f_{q}= & \frac{j !}{q !(j-q) !} P_{n}^{(q)}+\frac{j !}{(q-1) !(j+1-q) !} P_{n-1}^{(q-1)} \\
& +\sum_{r=1}^{q-1} \frac{j !}{(q-r-1) !(j+r+1-q) !} P_{n-r-1}^{(q-r-1)},
\end{aligned}
$$

where $2 \leqq q \leqq j, q \leqq n$.

Now $t=-j$ is by hypothesis a root of the associated system. So put $t=-j$ in (4) and after differentiation $q-r-1$ times substitute the resulting expression in (48), and simplify. This gives, for $q \leqq j, 2 \leqq q \leqq n$,

$$
\begin{aligned}
f_{q}= & \frac{j !}{q !(j-q) !} P_{n}^{(q)}+\frac{j !}{(q-1) !(j+1-q) !} P_{n-1}^{(q-1)} \\
& +j(j-1) P_{n}^{(q)} \sum_{r=1}^{q-1} \frac{(-1)^{r} r(j+r-1) !}{(q-r-1) !(j+r+1-q) !(r+1) !} \\
& +j P_{n-1}^{(q-1)} \sum_{r=1}^{q-1} \frac{(-1)^{r}(j+r-1) !}{(q-r-1) !(j+r+1-q) ! r !} .
\end{aligned}
$$

Now, 


$$
u^{j-2}(1-u)^{q}=\sum_{k=0}^{q} \frac{(-1)^{k} q ! u^{k+\jmath-2}}{(q-k) ! k !} .
$$

If (50) is differentiated $q-2$ times using the series the result is

$$
\frac{d^{q-2}}{d u^{q-2}}\left\{u^{j-2}(1-u)^{q}\right\}_{u=1}=\sum_{k=0}^{q} \frac{(-1)^{k} q !(j+k-2) !}{(q-k) ! k !(j+k-q) !} .
$$

On the other hand if (50) is differentiated $q-2$ times using the left member and Leibnitz rule, the resulting expression vanishes for $u=1$. So

$$
\sum_{k=0}^{q} \frac{(-1)^{k} q !(j+k-2) !}{(q-k) ! k !(j+k-q) !}=0
$$

Also

$$
u^{j-1}\left[-q(1-u)^{q-1}\right]=\sum_{k=1}^{q} \frac{(-1)^{k} q ! k u^{k+j-2}}{(q-k) ! k !} .
$$

As before, differentiating (53) $q-2$ times by the two methods and putting $u=1$ yields

$$
\sum_{k=1}^{q} \frac{(-1)^{k} q ! k(j+k-2) !}{(q-k) ! k !(j+k-q) !}=0 .
$$

Subtraction of (52) from (54) gives

$$
\sum_{k=2}^{q} \frac{(-1)^{k} q !(k-1)(j+k-2) !}{(q-k) ! k !(j+k-q) !}=\frac{(j-2) !}{(j-q) !} .
$$

Putting $k=r+1$ in this sum shows that the coefficient of $P_{n}^{(q)}$ in (49) is zero, while removal of the first term in (54) followed by the same index shift shows that the coefficient of $P_{n-1}^{(q-1)}$ in (49) is also zero. Thus $f_{q}=0$ if $q \leqq j$.

Now suppose $q>j$. In (47) again put $i=q-k$ in the summand. In this case, however, the sum is from $k=q-j$ to $k=q$. Then put $k=m+q-j$ in the resulting sum to obtain

$$
f_{q}=\sum_{m=0}^{j} \frac{j !}{(j-m) ! m !} P_{n+j-q-m}^{(j-m)}, j<q, 2 \leqq q<n .
$$

Since $t=-j$ is a root of the associated system, (4) becomes

$$
P_{n-r-1} \equiv \frac{(-1)^{r}(j+r-1) !}{(j-1) ! r !}\left\{P_{n-1}^{(r)}+\frac{r(j-1)}{(r+1)} P_{n}^{(r+1)}\right\},
$$

which in this form is valid also for $r=0$. In (57) put $r=m+q-j-1$, 
differentiate $j-m$ times, and substitute the resulting expression into (56). This will give, by rearranging,

$$
f_{q}=\frac{(-1)^{q-j-1}}{(j-1) !} P_{n-1}^{(q-1)} \sum_{m=0}^{j} \frac{j !(-1)^{m}}{(j-m) ! m !} \frac{(m+q-2) !}{(m+q-j-1) !}
$$

$$
+\frac{(-1)^{q-j-1}(j-1)}{(j-1) !} P_{n}^{(q)} \sum_{m=0}^{j} \frac{j !(-1)^{m}}{(j-m) ! m !} \frac{(m+q-j-1)(m+q-2) !}{(m+q-j) !} .
$$

Paralleling the previous case,

$$
0=\frac{d^{j-1}}{d u^{j-1}}\left\{u^{q-2}(1-u)^{j}\right\}_{u=1}=\sum_{m=0}^{j} \frac{j !(-1)^{m}}{(j-m) ! m !} \frac{(m+q-2) !}{(m+q-j-1) !}
$$

so the coefficient of $P_{n-1}^{(q-1)}$ in (58) is zero. If $j=1$ the second term of (58) is obviously zero. For $j>1$,

$$
0=\frac{d^{j-2}}{d u^{j-2}}\left\{u^{q-2}(1-u)^{j}\right\}_{u=1}=\sum_{m=0}^{j} \frac{j !(-1)^{m}(m+q-2) !}{(j-m) ! m !(m+q-j) !}
$$

and

(61) $0=\frac{d^{j-2}}{d u^{j-2}}\left\{u^{q-1}\left[-j(1-u)^{j-1}\right]\right\}_{u=1}=\sum_{m=0}^{j} \frac{j !(-1)^{m} m(m+q-2) !}{(j-m) ! m !(m+q-j) !}$.

If (60) is multiplied by $(q-j-1)$ and added to (61) it is seen that the coefficient of $P_{n}^{(q)}$ in (58) is also zero. Hence for all $q=2,3, \cdots, n, f_{q}=0$. So from (46), if $y(x)$ is any solution of (3) and the associated system has the negative integer root $t=-j$ then $y^{(n+j-1)}$ satisfies

$$
P_{n} z^{\prime}+\left(P_{n-1}+j P_{n}^{\prime}\right) z=R^{(j)} .
$$

Thus for some constant $k$ and appropriate choices of constants of integration,

$$
\frac{d^{n+j-1} y}{d x^{n+j-1}}=g(-j, x)\left\{k+\int\left[R^{(j)} / g(-j+1, x)\right] d x\right\},
$$

and the proof is complete. It is interesting to compare (62) with (12) and (63) with (18) and (34).

For the case of the second-order equation some results similar to the conclusion of Theorem 5 have been obtained by Abbé Lainé [18], though his results are in a considerably different form.

Example 5. The Hermite equation (32) has the associated root $t=-k$ so every solution can be obtained from

$$
y(x)=C \iint \cdots \int \exp \left(x^{2}\right)(d x)^{k+1}
$$


for some choice of $C$ and the constants of integration.

Though it has been assumed throughout that $x$ is a real variable the results can be easily extended to a complex variable setting.

In conclusion we state without proof the well-known result of applying the Euler transformation to (3) to obtain the Jordan-Pochhammer integrals. The theorem is given here in slightly different (but equivalent) form from the usual statement for reasons of comparison with the first solution of Theorem 1 and with Theorem 5 (see [19, pp. 104-111]).

THEOREM 6. If $R(x) \equiv 0$ and $a$ is a root of the associated system, then

$$
y(x)=\int_{C} \frac{g(a, t) d t}{(t-x)^{a-n+2}}
$$

is a solution to (3) provided that $C$ is any contour on any Riemann surface of the integrand for which

$$
h(x, t)=\frac{g(a+1, t)}{(t-x)^{a+1}}
$$

resumes its original value after describing $C$, and provided that differentiation with respect to $x$ under the integral sign is valid for $C$.

Proof. The proof of this theorem in an equivalent form is given in many of the standard references for differential equations. The usual approach is to use (7), instead of (3) with the additional condition (4).

It is, however, an interesting exercise in Taylor's formula and series manipulations to prove Theorem 6 by substituting (65) into (3) and using (4) to arrive at

$$
L[y]=-a(a-1) \cdots(a-n+2) \int_{C} \frac{\partial}{\partial t}\{h(x, t)\} d t .
$$

A proof of Theorem 6 by this method is given in detail in [3].

\section{REFERENCES}

1. James M. Horner, A note on the derivation of Rodrigues' formulae, Amer. Math. Monthly 70 (1963), 81-82.

2. Generalizations of the formulas of Rodrigues and Schläfli, Amer. Math. Monthly 71 (1964), 870-876.

3. - Generalizations of the formulas of Rodrigues and Schläfli, MA Thesis, University of Alabama, University, Alabama, August, 1962.

4. E. Kamke, Differentialgleichungen: Lösungsmethoden und Lösungen, Vol. 1, 4th ed., Akademische Verlagsgesellschaft, Leipzig, 1951.

5. E. L. Ince, Ordinary differential equations, Dover, New York, 1956.

6. A. R. Forsyth, Theory of differential equations. III (Ordinary linear equations), Vol. 4, Cambridge University Press, Cambridge, 1902.

7. M. C. Jordan, Cours d'analyse, Vol. III, 2nd ed., Gauthier-Villars, Paris, 1896. 
8. L. Pochhammer, Ueber ein Integral mit doppeltem Umlauf, Math. Ann. 35 (1890), 470-494.

9. _ Zier Theorie der Euler'schen Integrale, Math. Ann. 35 (1890), 495-526.

10. - Ueber eine Klasse von Integralen mit geschlossener Integrationscurve, Math.

Ann. 37 (1890), 500-543.

11. Ralph Palmer Agnew, Differential equations, McGraw-Hill, New York, 1942.

12. Murray R. Spiegel, Applied differential equations, Prentice-Hall, Englewood Cliffs, N. J., 1958.

13. Wilfred Kaplan, Advanced calculus, Addison-Wesley, Reading, Mass., 1952.

14. W. C. Brenke, On polynomial solutions of a class of linear differential equations of second order, Bull. Amer. Math. Soc. 36 (1930), 77-84.

15. N. Abramescu, Sulle serie di polinomi di una variable complessa. Le serie di Darboux, Ann. Mat. Pura Appl. (3) 31 (1922), 207-249.

16. E. T. Whittaker and G. N. Watson, Modern analysis, 4th ed., Cambridge University Press, Cambridge, 1927.

17. Earl D. Rainville, Special functions, Macmillan, New York, 1960.

18. Abbé Lainé, Sur l'integration de quelques équations différentielles de second ordre, Enseignement Math. 23 (1923), 163-173.

19. E. G. C. Poole, Linear differential equations, Oxford University Press, Oxford, 1936.

UNIVERSITY OF ALABAMA,

UNIVERSITY, Alabama 